\title{
Thermal axion cosmology and inflationary freedom
}

\author{
Eleonora Di Valentino* \\ Institut d'Astrophysique de Paris (UMR7095: CNRS \& UPMC- Sorbonne Universities), \\ F-75014, Paris, France \\ E-mail: valentin@iap.fr
}

In the primordial Universe the axion particles, which solve in an elegant way the CP problem in QCD, can be produced both thermally, contributing to the hot dark matter of the Universe, or not thermally, contributing to the cold dark matter. The thermal axions have masses strongly degenerate with those of the three active neutrinos, as they leave identical signatures in the different cosmological observables. In addition, while still relativistic states, they also contribute to the relativistic degrees of freedom, contributing to the dark radiation. Here, we will focus on the recent constraints from cosmology for the thermal axion mass, in the standard cosmological scenario $\Lambda \mathrm{CDM}$ as well as his extensions, but also taking into account the possibility that the primordial power spectrum could assume a more general shape than the usual power law description.

Neutrino Oscillation Workshop

4 - 11 September, 2016

Otranto (Lecce, Italy)

${ }^{*}$ Speaker. 


\section{Introduction}

The axion field arises as a solution to solve the strong CP problem in Quantum Chromodynamics $[1,2,3]$. The axion is the Pseudo-Nambu-Goldstone associated to a new global $U(1)_{P Q}$ (Peccei-Quinn) symmetry that, is spontaneously broken at an energy scale $f_{a}$. Here, we shall focus on the thermal axion scenario. In order to compute the present thermal axion relic density, the most relevant process is the axion-pion interaction, $\pi+\pi \rightarrow \pi+a$. The characteristic parameter for the thermal axion is $f_{a}$, the axion coupling constant, that can be related to the axion mass by

$$
m_{a}=\frac{f_{\pi} m_{\pi}}{f_{a}} \frac{\sqrt{R}}{1+R}=0.6 \mathrm{eV} \frac{10^{7} \mathrm{GeV}}{f_{a}},
$$

where the up-to-down quark masses ratio is taken as $R=0.553 \pm 0.043$, and $f_{\pi}=93 \mathrm{MeV}$ is the pion decay constant.

Thermal axions, while still relativistic, will increase the amount of radiation in the universe, contributing to the effective number of relativistic degrees of freedom $N_{\text {eff. }}$ In the standard cosmological $\Lambda$ CDM model with three active neutrino species, we expect $N_{\text {eff }}=3.046[4,5]$, where the 0.046 takes into account corrections for the non-instantaneous neutrino decoupling from the primordial plasma. An extra $\Delta N_{\text {eff }}=N_{\text {eff }}-3.046$ modifies the damping tail of the Cosmic Microwave Background (CMB) temperature angular power spectrum, changing two important scales at recombination, the sound horizon and the Silk damping, as well as also the primordial abundances of the light elements predicted by Big Bang Nucleosynthesis. When thermal axions become nonrelativistic particles, they will affect the different cosmological observables in an analogous way to that of massive neutrinos, i.e. by increasing the amount of the (hot) dark matter density in our universe. Axions will suppress the structure formation at scales smaller than its free-streaming scale, favouring clustering only at large scales. Thermal axions will also leave an imprint on the CMB temperature anisotropies, via the early integrated Sachs-Wolfe effect. For the details, we refer the reader to Ref. [6].

\section{Results}

In light of the Planck 2015 temperature and polarization data [7, 8, 9], we computed the new bounds on the thermal axion mass and on the effective number of relativistic species $N_{\text {eff }}$, including in both the cases the massive neutrinos. For the details regarding the method and the combination of datasets used, we refer the reader to Refs. [10, 11, 12].

In Table IV of [10] we report the 95\% CL constraints on the cosmological parameters from some of the different combinations of data sets explored in the $\Lambda \mathrm{CDM}+\sum m_{v}+N_{\text {eff }}$ model, focusing on the effect of the $H_{0}$ prior. Concerning the effective number of relativistic species $N_{\text {eff }}$, the addition of Planck polarisation measurements leads to a major improvement in its 95\% CL bound, which becomes more robust and almost independent of the external priors. The constraints on the total neutrino mass are less restrictive in this more general scenario, given the strong degeneracy between $\sum m_{v}$ and $N_{\text {eff }}$ : a larger matter density can be compensated with an extra radiation component, and, consequently, $\sum m_{v}$ and $N_{\text {eff }}$ are positively correlated.

Table II of [11] shows the 95\% CL constraints on the parameters of the mixed hot dark matter scenario explored, with both axions and neutrinos as extra hot thermal relics, for the different 
combinations of cosmological data sets. Notice that Planck temperature and polarisation measurements set 95\% CL upper bounds of $\sum m_{v}<0.441 \mathrm{eV}$ and $m_{a}<2.09 \mathrm{eV}$ respectively. When Planck CMB lensing constraints [13] are considered, the neutrino mass bounds is pulled away from zero, and we obtain $\sum m_{v}<0.538 \mathrm{eV}$ and $m_{a}<1.67 \mathrm{eV}$ at $95 \% \mathrm{CL}$. The largest impact on both $\sum m_{v}$ and $m_{a}$ bounds comes from the large scale structure information as well as from the prior on $H_{0}$ from the Hubble Space Telescope (HST) experiment [14]. Concerning the $H_{0}$ prior, the 95\% CL upper bounds on the thermal relic masses become $\sum m_{v}<0.182 \mathrm{eV}$ and $m_{a}<1.19 \mathrm{eV}$. The reason for this large improvement is due to the large degeneracy between $\sum m_{v}$ and $H_{0}$. However, the tightest axion and neutrino mass constraints arise when are added Baryon Acoustic Oscillation (BAO) data [15, 16, 17], and we find a similar effect while the full-shape data come from only one survey, the WiggleZ survey [18]. Using the full matter power spectrum measurements from the former experiment, we obtain 95\% CL upper bounds of $\sum m_{v}<0.291 \mathrm{eV}$ and $m_{a}<0.835 \mathrm{eV}$. The 95\% CL upper bound of $\sum m_{v}<0.156 \mathrm{eV}$ for Planck TT, TE, EE+ lowP and BAO combination is very close to the one quoted by the Planck collaboration for the same data sets, $\sum m_{v}<0.17 \mathrm{eV}$ [8]. However, our constraint is tighter, as we are also considering axions as additional thermal relics, and there exists a strong degeneracy among these $\sum m_{v}$ and $m_{a}$. The addition of the BAO and the HST datasets leads to the stronger constraint on the neutrino mass to date in the linear perturbation regime, $\sum m_{v}<0.136 \mathrm{eV}$ at $95 \% \mathrm{CL}$. The corresponding bound on the axion mass is $m_{a}<0.709 \mathrm{eV}$.

In [12] we relax the assumption on the usual simple power-law description for the primordial perturbations, governed by an amplitude and a scalar spectral index, and we constrain the mass of the thermal axion and the number of relativistic degrees of freedom using a non-parametric description of the Primordial Power Spectrum (PPS) of the scalar perturbations, in order to test the robustness of these bounds. In particular, we decided to use a function to interpolate the PPS values in a series of nodes at fixed position, named "piecewise cubic Hermite interpolating polynomial" (PCHIP) [19]. We use the modified version detailed in Gariazzo et al. 2014 [20].

In Table 6 of [12], we can see that, once the freedom in the PPS is introduced, a strong degeneracy between the PCHIP nodes $P_{s, j}$ and $N_{\text {eff }}$ appears. Even if the constraints on $N_{\text {eff }}$ are loosened for the PCHIP PPS case, all the dataset combinations give constraints on $N_{\text {eff }}$ that are compatible with the standard value 3.046 at $95 \%$ CL. The mild preference for $N_{\text {eff }}>3.046$ arises mainly as a volume effect in the Bayesian analysis, since the PCHIP PPS parameters can be tuned to reproduce the observed CMB temperature spectrum for a wide range of $N_{\text {eff }}$ values. As expected, the degeneracy between the nodes $P_{s, j}$ and $N_{\text {eff }}$ shows up at high wavemodes, where the Silk damping effect is dominant.

The results obtained with the inclusion of the full CMB polarization data are shown in Table 7 of [12]. The introduction of the polarization data helps in improving the constraints in the models with a PCHIP PPS, since the effects of increasing $N_{\text {eff }}$ and changing the PPS are different for the temperature-temperature, the temperature-polarization and the polarization-polarization correlation spectra. When the degeneracies are broken, the preferred value of $N_{\text {eff }}$ is very close to the standard value 3.046. Apparently, the Planck polarization data seem to prefer a value of $N_{\text {eff }}$ slightly smaller than 3.046 for all the datasets except those including the BAO data, but the effect is not statistically significant.

In Table 10 of [12] we can see that the bounds on the axion mass are relaxed when considering 
the PCHIP PPS with respect to the power-law PPS. In addition, in this case, we can also notice a weakening of the total neutrino mass constraints. The only exception is when considering the BAO measurements, since they are directly sensitive to the free-streaming nature of the two relic particles. Concerning the CMB measurements only, the Planck TT+lowP data are not able to constrain the axion mass in the PCHIP approach, providing $\Sigma m_{v}<2.20 \mathrm{eV}$ at $95 \% \mathrm{CL}$ versus the $\Sigma m_{v}<0.616 \mathrm{eV}$ at $95 \% \mathrm{CL}$ limit obtained for the power-law approach. The most stringent bounds arise when using the BAO data in both parameterizations: we have $m_{a}<1.03 \mathrm{eV}$ at $95 \% \mathrm{CL}$ and $\Sigma m_{v}<0.205 \mathrm{eV}$ at $95 \% \mathrm{CL}$ in the PCHIP case. Finally, when considering the lensing dataset with the PCHIP PPS, we obtain $m_{a}<2.13 \mathrm{eV}$ at $95 \% \mathrm{CL}$ and $\Sigma m_{v}<1.42 \mathrm{eV}$ at $95 \% \mathrm{CL}$.

Table 11 of [12] shows the bounds on the axion and total neutrino masses, when adding the Planck polarization data. The axion (neutrino) mass constraints are, in general, slightly weaker (stronger) than those obtained using only the temperature power spectrum at small angular scales. In particular, focusing on the CMB measurements alone, the Planck TT,TE,EE+lowP data provides upper limits on the thermal axion mass and on the neutrino masses in the PCHIP approach of $m_{a}<2.37 \mathrm{eV}$ at $95 \% \mathrm{CL}$ and $\Sigma m_{v}<0.718 \mathrm{eV}$ at $95 \% \mathrm{CL}$, respectively. The most stringent bounds on both the axion mass and on the total neutrino mass arise, as usual, from the addition of BAO data. We find $m_{a}<1.18 \mathrm{eV}$ at $95 \% \mathrm{CL}$ and $\Sigma m_{v}<0.180 \mathrm{eV}$ at $95 \% \mathrm{CL}$ in the PCHIP PPS, respectively. Finally, when considering the lensing dataset within the PCHIP PPS we obtain $m_{a}<2.33 \mathrm{eV}$ at 95\% CL and $\Sigma m_{v}<0.936 \mathrm{eV}$ at $95 \% \mathrm{CL}$.

\section{Conclusions}

Thermal axions can contribute to the hot dark matter component of the universe, together with the three active neutrinos. Axions have a free-streaming nature, suppressing structure formation at small scales, and also contribute to the dark radiation background, leading to deviations of the relativistic degrees of freedom $N_{\text {eff }}$ from its canonically expected value 3.046.

The neutrino effective number provided by the Planck data is totally consistent with its standard value 3.046. Anyway, there is still the possibility to have some relic components. We constrained $N_{\text {eff }}$ also using an alternative, non-parametric description of the primordial power spectrum of the scalar perturbations, and we find that with the introduction of the polarization data, the PCHIP parametrization of the PPS does not favor anymore, with respect to the temperature only data, an extra dark radiation. The effective neutrino number is perfectly in agreement with the Planck collaboration bounds. We obtain a robust 95\% CL range of $2.5<N_{\text {eff }}<3.5$, with very small differences in both the central values and allowed ranges for the several data combinations and PPS approaches.

In order to test the robustness of the cosmological axion mass bounds, we constrained also it using the PCHIP parametrization of the PPS and tightest bound we find has been obtained when considering BAO measurements together with Planck TT+lowTEB, with $m_{a}<1.03 \mathrm{eV}$ at $95 \%$ CL. In the standard power-law primordial power spectrum modeling, the same combination of data gives $m_{a}<0.939 \mathrm{eV}$ at $95 \% \mathrm{CL}$. The axion mass constraints are only marginally affected from the change in the PPS. 


\section{References}

[1] R. D. Peccei and H. R. Quinn, Phys. Rev. Lett. 38, 1440 (1977); R. D. Peccei and H. R. Quinn, Phys. Rev. D 16, 1791 (1977).

[2] S. Weinberg, Phys. Rev. Lett. 40, 223 (1978).

[3] F. Wilczek, Phys. Rev. Lett. 40, 279 (1978).

[4] G. Mangano, G. Miele, S. Pastor, T. Pinto, O. Pisanti and P. D. Serpico, Nucl. Phys. B 729, 221 (2005) [hep-ph/0506164].

[5] P. F. de Salas and S. Pastor, JCAP 1607, no. 07, 051 (2016) [arXiv:1606.06986 [hep-ph]].

[6] E. Giusarma et al., Phys. Rev. D 90, 043507 (2014)

[7] R. Adam et al. [Planck Collaboration], arXiv:1502.01582 [astro-ph.CO].

[8] P. A. R. Ade et al. [Planck Collaboration], Astron. Astrophys. 594, A13 (2016) [arXiv:1502.01589 [astro-ph.CO]].

[9] N. Aghanim et al. [Planck Collaboration], Astron. Astrophys. 594, A11 (2016) [arXiv:1507.02704 [astro-ph.CO]].

[10] E. Di Valentino, E. Giusarma, O. Mena, A. Melchiorri and J. Silk, Phys. Rev. D 93, no. 8, 083527 (2016) [arXiv:1511.00975 [astro-ph.CO]].

[11] E. Di Valentino, E. Giusarma, M. Lattanzi, O. Mena, A. Melchiorri and J. Silk, Phys. Lett. B 752, 182 (2016) [arXiv:1507.08665 [astro-ph.CO]].

[12] E. Di Valentino, S. Gariazzo, M. Gerbino, E. Giusarma and O. Mena, Phys. Rev. D 93, no. 8, 083523 (2016) [arXiv:1601.07557 [astro-ph.CO]].

[13] P. A. R. Ade et al. [Planck Collaboration], Astron. Astrophys. 594, A15 (2016) [arXiv:1502.01591 [astro-ph.CO]].

[14] A. G. Riess et al., Astrophys. J. 730, 119 (2011) Erratum: [Astrophys. J. 732, 129 (2011)] [arXiv:1103.2976 [astro-ph.CO]].

[15] F. Beutler et al., Mon. Not. Roy. Astron. Soc. 416, 3017 (2011).

[16] A. J. Ross, L. Samushia, C. Howlett, W. J. Percival, A. Burden and M. Manera, Mon. Not. Roy. Astron. Soc. 449 (2015) no.1, 835 [arXiv:1409.3242 [astro-ph.CO]].

[17] L. Anderson et al. [BOSS Collaboration], Mon. Not. Roy. Astron. Soc. 441, 24 (2014).

[18] D. Parkinson et al., Phys. Rev. D 86, 103518 (2012) [arXiv:1210.2130 [astro-ph.CO]].

[19] F. Fritsch and R. Carlson, SIAM Journal on Numerical Analysis, 17, 238 (1980).

[20] S. Gariazzo et al., arXiv:1412.7405 [astro-ph.CO]. 